\title{
An Equation of State Unifies Diversity, Productivity, Abundance and Biomass
}

\author{
John Harte ( $\sim$ jharte@berkeley.edu ) \\ University of California, Berkeley \\ Micah Brush \\ University of Alberta
}

Erica Newman

University of Arizona

Kaito Umemura

Kobe University

\section{Article}

Keywords:

Posted Date: March 2nd, 2022

DOI: https://doi.org/10.21203/rs.3.rs-1362894/v1

License: (c) (1) This work is licensed under a Creative Commons Attribution 4.0 International License.

Read Full License

Version of Record: A version of this preprint was published at Communications Biology on August 25th, 2022. See the published version at https://doi.org/10.1038/s42003-022-03817-8. 
An Equation of State Unifies Diversity, Productivity, Abundance and Biomass

Authors: John Harte ${ }^{1,2,3^{*}}$, Micah Brush ${ }^{1,4}$, Erica A. Newman ${ }^{5}$, Kaito Umemura ${ }^{1,6}$

\author{
Affiliations: \\ ${ }^{1}$ The Energy and Resources Group, University of California; Berkeley, CA 94720, USA \\ 2 The Rocky Mountain Biological Laboratory; Gothic, CO 81224, USA \\ 3 The Santa Fe Institute; Santa Fe, NM 87501, USA \\ ${ }^{4}$ The Department of Mathematical and Statistical Sciences, University of Alberta; \\ Edmonton, AB, T6G 2G1, CA \\ ${ }^{5}$ School of Natural Resources \& the Environment, University of Arizona; Tucson, AZ 85721 \\ ${ }^{6}$ Graduate School of Human Development and Environment, Kobe University; Kobe, Hyogo \\ *Corresponding author. Email: jharte@berkeley.edu
}

15 657-8501, Japan

To advance understanding of biodiversity and ecosystem function, ecologists seek widely applicable relationships among species diversity and other ecosystem characteristics such as species productivity, biomass, and abundance ${ }^{1-4}$. These metrics vary widely across ecosystems and no relationship among any combination of them that is valid across habitats, taxa, and spatial scales, has heretofore been found. Here we derive such a relationship, an equation of state, among species richness, energy flow, biomass and 25 abundance by combining results from the Maximum Entropy Theory of Ecology $y^{5-7}$ and the Metabolic Theory of Ecology ${ }^{8,9}$. It accurately captures the relationship among these state variables in 42 data sets, including vegetation and arthropod communities, that span a wide variety of spatial scales and habitats. The success of our ecological equation of state opens opportunities for predicting difficult-to-measure state variables from measurements of others, adds support for two current theories in ecology, and is a step toward unification in ecology. 
A major focus of ecology is the study of species diversity across ecosystems and its relationship to ecosystem structure and function ${ }^{1-4}$. Considerable effort has been directed at the challenge of finding other macro-level ecosystem variables, such as productivity that might correlate with and potentially explain the wide range of values of species richness observed in different habitats, climates, and taxa, across spatial scales. Diversity and productivity are system-level descriptors of ecosystems. Other system descriptors include the total biomass and abundance of individuals. No widely-observed relationship among any combination of these four system variables has heretofore been found.

10 Diversity, productivity, abundance and biomass in ecology are loosely analogous to state variables in thermodynamics, such as the pressure, volume, temperature and number of moles of a container of gas. In thermodynamics, an equation of state, or universal relationship among state variables, exists in the form of the ideal gas law: $P V=n R T$. Equations of state in physics and chemistry derive from fundamental theory, but in macroecological studies of ecosystems such a framing has been lacking. A successful equation of state derived from ecological theory would deepen our understanding of ecology, allow prediction of diversity or productivity from knowledge of other system-level state variables, and potentially enhance applications of ecological theory to conservation and restoration ${ }^{10}$.

20 Here we present, and show the empirical validity of, an equation of state in ecology that derives from combining ecological theory ${ }^{5-7}$ based on the maximum information entropy (MaxEnt) inference principle ${ }^{11,12}$ with a finding from metabolic scaling theory 8,9 .

In thermodynamics, it has proven useful to distinguish the micro-level and the macro-level descriptions of the system, and then maximize Shannon information entropy ${ }^{11,12}$ to infer phenomena at the micro-level, such as the Boltzmann distribution of molecular kinetic energies, from constraints imposed by state variables at the macro-level, such as total system energy and number of molecules. Extending this concept to ecology, we take the micro-level variables to be metabolic rates, $\varepsilon$, of individuals and abundances, $n$, of species within an ecological community 30 of, for example, plants, arthropods or mammals. We take the macro-level state variables to be the total number of species, $S$, total number of individuals, $N$, in the community, and total metabolic 
rate, $E$, of all the individuals in a given area $A$. An application of MaxEnt then results in the Maximum Entropy Theory of Ecology (METE) $)^{5-7}$.

At the core of METE is the "ecological structure function" $R(n, \varepsilon \mid S, N, E)$, a joint probability distribution over two micro-level variables, abundance $n$ and metabolism $\varepsilon$, that is constrained by values of the state variables $\mathrm{S}, N$ and $E . R \cdot d \varepsilon$ is the probability that if a species is picked from the species pool, then it has abundance $n$, and if an individual is picked at random from all the species with abundance $n$, then its metabolic energy requirement is in the interval $(\varepsilon, \varepsilon+\mathrm{d} \varepsilon)$. From the definition of $R$, total abundance, $N$, is $S$ times the average of $n$, and total metabolic rate, $E$, is $S$ times the average of $n \varepsilon$, where both averages are taken over the distribution $R$. The form of $R$ is derived by maximizing its Shannon information entropy subject to the constraints imposed by the two independent ratios that can be formed from $S, N$ and $E: N / S$ and $E / S$. The MaxEnt solution $^{5}$ is $R=e^{-\lambda_{1} n} e^{-\lambda_{2} n \varepsilon} / Z$ where the $\lambda_{i}$ are Lagrange multipliers and $Z$ is a normalizing factor, all of which depend only on $S, N$ and $E$.

From the ecological structure function, predictions for the distribution of abundances across species and the distribution of metabolic rates across individuals, as well as a relationship between the abundance of a species and the average metabolism of its individuals can be derived $^{5-7}$. In spatially explicit applications, the area of the system is included as a fourth state variable and additional predictions follow, including a universal scale-collapse expression for the species-area relationship ${ }^{13}$. If higher taxonomic levels such as genus or family are included as additional state variables in addition to species, then the theory predicts the distribution of species over these added taxonomic categories and an explicit dependence of the abundancemetabolism relationship on the taxonomic structure of the community ${ }^{14}$.

In METE, the three state variables $S, N$, and $E$ are assumed to be mutually independent, and empirically that assumption is supported (Appendix 1 in Supplementary materials, and Supplementary video 1). To derive an ecological analog of the thermodynamic equation of state, we introduce a fourth state variable, $B$, the total biomass in the community. Units are normalized 30 such that the smallest mass and the smallest metabolic rate within a censused plot are each assigned a value of 1 . 
The ecological analog of the thermodynamic equation of state, an expression for biomass, $B$, in terms of $S, N$, and $E$, arises if we combine METE with a scaling result from the metabolic theory of ecology $(\mathrm{MTE})^{8,9}$. In particular, we assume the MTE scaling relationship ${ }^{8}$ between the metabolic rate, $\varepsilon$, of an individual organism and its mass, $m: \varepsilon \sim m^{3 / 4}$. Because of our units convention, the proportionality constant in this scaling relationship can be assigned a value of 1 . From the definition of the structure function, it follows ${ }^{15}$ that averaging the biomass of individuals times the abundance of species, $n \varepsilon^{4 / 3}$, over the distribution $R$ and multiplying by the number of species gives the total ecosystem biomass as a function of $S, N$, and $E$. In other words:

$$
B=S \sum_{n} n \int d \varepsilon \varepsilon^{4 / 3} R(n, \varepsilon \mid S, N, E)
$$

Both the sum and integral in the above equation can be calculated numerically, and Python code to do so for a given set of state variables $S, N, E$, is available at github.com/micbru/equation_of_ 15 state/.

We can also approximate the solution analytically (see Materials and Methods in Supplementary Materials) if $E>>>>S>1$ :

$$
B=c \frac{E^{4 / 3}}{S^{1 / 3} \ln (1 / \beta)}
$$

where $c \approx(7 / 2) \Gamma(7 / 3) \approx 4.17$ and $\beta=\lambda_{1}+\lambda_{2}$ is estimated $^{6,16}$ from the relationship $\beta \ln (1 / \beta) \approx S / N$. This approximation is good to within $10 \%$ of the exact numerical calculation if $N / S$ is larger than a few hundred, and $E / N$ is larger than $~ 50$ (Fig. S1 in Supplementary 25 Materials, Materials and Methods) and can be made more accurate by multiplying by 1 $1.16 \beta^{1 / 3}$ (good to within $10 \%$ for $N / S$ and $E / N$ larger than about 10$)$.

Empirical values of $E$ and $B$ can be estimated from the same data. In particular, if measured metabolic rates of the individuals are denoted by $\varepsilon_{i}$, where $i$ runs from 1 to $N$, then $E$ is given by the sum over the $\varepsilon_{i}$ and $B$ is given by the sum over the $\varepsilon_{i}{ }^{4 / 3}$. Similarly, if the mass, $m_{i}$, of each 
individual is measured, then $B$ is the sum over the $m_{i}$ and $E$ is the sum over the $m_{i}^{3 / 4}$. In practice, for animal data, metabolic rate is often estimated by measuring mass and then using metabolic scaling, while for tree data metabolic rate is estimated from measurements of individual tree basal areas, which are estimators ${ }^{17}$ of the $\varepsilon_{i}$. Note that $B^{3 / 4}=\left(\sum \varepsilon_{i}{ }^{4 / 3}\right)^{3 / 4} \leq \sum \varepsilon_{i}=E$.

A test of Eq. 1 that compares observed and predicted values of biomass with data from 42 censused plots across a variety of habitats, spatial scales and taxa is shown in Fig. 1. The 42 plots are listed and described in Table S-2, Appendix 2, in Supplementary Materials. The communities censused include arthropods and plants, the habitats include both temperate and tropical, and the census plots range in area from 0.0064 ha to 50 ha. As seen in the figure, $99.4 \%$ of the variance in the observed values of $B$ is explained by the predicted values of $B$.

Fig. 2 addresses the possible concern that the success of Eq. 1 shown in Fig. 1 simply reflects approximate constancy of the ratio of $E$ (which is the sum of the individual metabolic rates) to 15 the three-quarters power of the total biomass, $B$ (which is sum of the four-thirds powers of the metabolic rates) across all the data sets. If the $E: B^{3 / 4}$ ratio were constant, then $S$ and $N$ would play no effective role in the equation of state. Variation in that ratio is predicted by Eq. 1 to depend upon $S$ and $N$. Eq 2 shows that dependence to be in the approximate combination $S^{1 / 4} \ln ^{3 / 4}(1 / \beta(N / S))$. In Fig. 2, the observed and predicted values of $E / B^{3 / 4}$ calculated from Eq. 1, 20 are compared, showing nearly four-fold variation in that ratio across the data sets. The equation of state predicts $60 \%$ of the variance in the ratio.

Total productivity of an ecological community and its relationship to diversity and other ecosystem characteristics is a focus of interest in ecology ${ }^{1}$. Interpreting total metabolic rate $E$ in

25 our theory as gross productivity, then in the limit $1<<S<<N<<E$, we can rewrite Eq. 2 to highlight the role of diversity and the other state variables in determining this quantity:

$$
E=c^{-3 / 4} S^{1 / 4} B^{3 / 4} \ln ^{3 / 4}(1 / \beta)
$$

30 Here, as shown in Eq. 2, $c$ is a universal constant ( 4.17). From Eq. 3, and the fact that up to a $\operatorname{logarithmic}$ correction $\beta$ varies as $S / N$, biomass exerts the strongest influence on $E$. With 
biomass fixed, productivity varies approximately as $S^{1 / 4}$, with a logarithmic correction, times $\ln ^{3 / 4}(N)$ with a $\log (\log (N))$ correction. Thus, dependence of productivity on abundance is weakest, on biomass is strongest, and on species richness is of intermediate strength. Testing these predictions requires finding communities with the same values of pairs of state variables, and thus it is most feasible to compile further tests of Eq. 1 by allowing all four variables to vary as in Figs. 1 and 2. We further note that if $1<<S<<N<<E$ we can re-express Eq. 3 entirely in terms of three ratios, $E / S, B / S$, and $N / S$, rather than the four state variables, and obtain:

$$
E / S=c^{-3 / 4}(B / S)^{3 / 4} \ln ^{3 / 4}(1 / \beta(N / S))
$$

To date, empirical cross-ecosystem surveys of relationships among community metabolism, biomass, species richness, and abundance have largely focused on uncontrolled pairwise relationships as, for example ${ }^{1}$, between productivity and biomass but without controlling for species richness and abundance, and found considerably more scatter in the relationship than is shown in Fig. 1. In Supplementary Materials, Appendix 1, pairwise comparisons among state variables are shown for the data sets used here, illustrating the absence of strong relationships among pairs of state variables when the remaining pair of state variables is unconstrained. The largest $R^{2}$ value in all comparisons is 0.987 , between $\ln (B)$ and $\ln (E)$, which is expected as they 20 are computed from the same data. Noting $(1-0.994) /(1-0.987) \approx 0.46$, the unexplained variance in the observed $\ln (B)$ using the equation of state is less than half that of the second-best predictor, $\ln (E)$. All other tested relationships shown in the Appendix were significantly worse.

Far more testing of the ecological equation of state is warranted. Moreover, Eq. 1 raises many new questions. As with other empirical successes of METE, it is unclear why an apparently mechanism-free theory should work at all in ecology. Our tentative answer is that in ecosystems in which the state variables are relatively constant in time, the myriad of mechanisms and traits, differing from organism to organism and species to species, confer upon them all sufficient fitness to co-exist ${ }^{18}$. In other words, just as in an ideal gas where interactions among molecules 30 can be ignored in thermodynamic equilibrium except under extreme conditions, so the mechanisms operating in ecosystems can be ignored because they simply represent different 
tactics for achieving a common goal: sufficient fitness.

Pursuing that idea, we might more generally expect the predictions of a top-down MaxEnt approach to fail under ecological disturbances that sufficiently alter the fitness landscape, resulting in dynamic, not static, state variables. This indeed appears to be the case for METE's prediction of the species-abundance distribution (SAD) and the species-area relationship (SAR), as discussed elsewhere $7,15,34,35,38$. An implication of this is that a survey of highly disturbed ecosystems with transitioning state variables, might reveal significant deviations from Eq. 1, which contains no dynamics. As in thermodynamics, where failure of the ideal gas law under extreme values of the state variables revealed the existence of van der Waals forces between polar molecules, so different types of failure of Eq. 1 in disturbed ecosystems might shed light on the nature of disturbance, resilience of the current state, and prospects for recovery ${ }^{15}$.

Applying those insights to our equation of state, four data points stand out as outliers in Fig. 1 and especially Fig. 2, These are the four temperate tree communities (blue triangles). One of the common characteristics of these four sites is that they are currently undergoing secondary succession processes from disturbances over the past decades to centuries ${ }^{28,30-32}$. Moreover, they stand out from the other sites by their relatively small $S$-to $N$ ratio. A more thorough survey of the validity of Eq. 1 across a spectrum of levels and kinds of disturbance is suggested.

We note that ecosystem area does not appear explicitly in Eq. 1; area only enters implicitly through the area-dependence of the state variables. At least over the range of areas spanned by our data sets ( $\left.50 \mathrm{ha} / 0.0064 \mathrm{ha} \sim 10^{4}\right)$, this absence of explicit area-dependence in our equation of state is validated. However, just as some of METE's other predictions fail at large regional 25 scales $^{6}$, we expect to see significant deviations from the predicted equation of state at very large spatial scales.

The full mass-metabolism scaling relationship ${ }^{9}$ includes a temperature-dependent multiplicative term such that $\varepsilon \sim e^{-E_{0} / k T} m^{3 / 4}$, where $E_{0}$ is an activation energy, $k$ is Boltzmann's constant, and $30 T$ is temperature in Kelvin. The effect of this temperature correction on the accuracy of the equation of state remains to be tested. 
We have used a metabolic scaling exponent of 3/4 in all of the above, but there is both controversy over, and empirical variability in, its actual value ${ }^{39}$. If the scaling exponent is taken to be $2 / 3$, as can be obtained from an energy budget model in which energy loss is proportional to the surface area of an organism ${ }^{40}$ then the exponent of 4/3 in Eq. 2 becomes 3/2, and a different form of the equation of state is obtained. Moreover, the empirical value of $E$ calculated from measured values of the masses of individuals will differ and thus the empirical ratio of $E$ to $B$ will differ. In Appendix 3 of Supplementary Materials, we show that the empirical validity of the equation of state is reduced if the metabolic scaling exponent is assumed to be $2 / 3$ instead of 3/4. In particular for the test shown in Fig. 1, the $R^{2}$ value drops from 0.994 to 0.986 , and for Fig. 2 , the $R^{2}$ drops from 0.600 to 0.477 . On the other hand, if the metabolic scaling exponent is 1 , then with our units convention, empirically $E=B$ and Eq. 1 predicts exactly that. An example of a community in which $E \approx B$ is a microbiome in which the masses of bacteria differ from one another by a much smaller factor than, for example, the masses of trees in a forest differ from one another. It is noteworthy that for microorganisms, there is evidence ${ }^{9}$ that the metabolic scaling exponent is in fact closer to 1 than to $3 / 4$.

Ecosystems exhibit numerous idiosyncratic phenomena, but ubiquitous patterns nevertheless exist. The latter motivates the search for general laws. We have provided evidence here for the validity of one such law, an ecological equation of state, which can be derived by combining two ecological theories, METE and MTE. Each of these theories had previously been shown to have broad explanatory power, and our result demonstrates the utility of combining theories that in combination yield more than the sum of the parts ${ }^{41}$. Our ecological equation of state is a simple property of complex ecosystems, and it appears to be valid across spatial scales, across types of

25 habitat, and across different taxonomic groups. Parallel advances in our understanding of relationships among macro-scale variables in other types of complex systems, such as economies $^{42}$ or networks ${ }^{43,44}$ may also be possible by combining the powerful MaxEnt inference procedure with appropriate scaling laws. 


\section{References}

1. Jenkins, D. G. Estimating ecological production from biomass. Ecosphere. 6, 1-31 (2015).

2. Currie, D.J., 1991. Energy and large-scale patterns of animal-and plant-species richness. The American Naturalist, 137(1), 27-49 (1991).

10

3. O'Connor, M. I., Gonzalez, A., Byrnes, J. E. K., Cardinale, B. J., Duffy, J. E., Gamfeldt, L., Griffin, J. N., Hooper, D., Hungate, B. A., Paquette, A., Thompson, P. L., Dee, L. E., \& Dolan, K. L. A general biodiversity-function relationship is mediated by trophic level. Oikos 126(1), 18-31 (2017). https://doi.org/10.1111/oik.03652

15

4. Gillman, L. N., Wright, S. D., Cusens, J., McBride, P.D., Malhi, Y., Whittaker, R. J., Latitude, productivity and species richness. Glob. Ecol. Biogeogr. 24, 107-117 (2015).

5. Harte, J., Zillio, T., Conlisk, E., Smith, A. B. Maximum entropy and the state-variable approach to macroecology. Ecology 89, 2700-2711 (2008).

6. Harte, J. Maximum Entropy and Ecology: A Theory of Abundance, Distribution, and Energetics. OUP Oxford (2011).

25 7. Harte, J., Newman, E. A. Maximum information entropy: a foundation for ecological theory. Trends Ecol. Evol. 29, 384-389 (2014).

8. West, G. B., Brown, J. H., Enquist B. J. A general model for the origin of allometric scaling laws in biology. Science 276, 122-126 (1997). 
9. Brown, J. H., Gillooly, J. F., Allen, A. P., Savage, V. M., West, G. B. Toward a metabolic theory of ecology. Ecology 85, 1771-1789 (2004).

10. Santini, L., Antão, L. H., Jung, M., Benítez-López, A., Rapacciuolo, G., Di Marco, M., Jones, F. A. M., Haghkerdar, J. M., González-Suárez, M. The interface between Macroecology and Conservation: existing links and untapped opportunities. Front. Biogeogr. (2021), doi:10.21425/F5FBG53025. (1957).

12. Jaynes, E. T. On the rationale of maximum-entropy methods. Proc. IEEE. 70, 939-952 (1982).

15

13. Harte, J., Smith, A. B., Storch, D. Biodiversity scales from plots to biomes with a universal species-area curve. Ecol. Lett. 12, 789-797 (2009).

14. Harte, J., Rominger, A., Zhang, W. Integrating macroecological metrics and community taxonomic structure. Ecol. Lett. 18, 1068-1077 (2015).

15. Harte, J., Umemura, K. Brush, M. DynaMETE: a hybrid MaxEnt-plus-mechanism theory of dynamic macroecology. Ecol. Lett. 24, 935-949 (2021). theory of ecology. Entropy (2019) (https:/www.mdpi.com/1099-4300/21/7/712). 
17. Niklas, K. J., Enquist, B. J. Invariant scaling relationships for interspecific plant biomass production rates and body size. Proc. Natl. Acad. Sci. U. S. A. 98, 2922-2927 (2001).

18. Bradford, M. G., Murphy, H. T., Ford, A. J., Hogan, D., Metcalfe, D. J. CSIRO Permanent Rainforest Plots of North Queensland (2014).

19. Bradford, M. G., Murphy, H., Ford, A., Hogan, D., Metcalfe, D. CSIRO Permanent Rainforest Plots of North Queensland. v3. (2014). CSIRO. Data Collection. https://doi.org/10.4225/08/59475c67be7a4

10

20. Hubbell, S. P., Foster, R. B., O’Brien, S. T., Harms, K. E., Condit, R., Wechsler, B., Wright, S. J., de Lao S. L. Light-Gap disturbances, recruitment limitation, and tree diversity in a neotropical forest. Science 283, 554-557 (1999).

21. Condit, R. Tropical Forest Census Plots: Methods and Results from Barro Colorado Island, Panama and a Comparison with Other Plots. Springer Science \& Business Media (1998).

22. Condit, R. Pérez, R., Aguilar, S., Lao, S., Foster, R., Hubbell, S. Complete data from the Barro Colorado 50-ha plot: 423617 trees, 35 years. URL https://doi.org/10.15146/5xcp-0d46 (2019).

23. Condit, R., Aguilar, S., Hernandez, A., Perez, R., Lao, S., Angehr, G., Hubbell, S. P., Foster, R. B. Tropical forest dynamics across a rainfall gradient and the impact of an El Niño dry season. J. Trop. Ecol. 20, 51-72 (2004). 
24. Selhorst, D., Brown, I. F.. LBA-ECO LC-02 Biophysical Measurements of Forests, Acre, Brazil: 1999-2002. ORNL DAAC (2014) (https://daac.ornl.gov/cgibin/download.pl?ds_id=1237\&source=schema_org_metadata).

25. Lourenço Jr. J., Newman E. A., Ventura J. A., Milanez C. R., Thomaz L. D., Wandekoken D. T., Enquist B. J. Soil-associated drivers of plant traits and functional composition in Atlantic Forest coastal tree communities. Ecosphere 12(7): e03629 (2021).

26. Munger, W., Wofsy, S. Biomass Inventories at Harvard Forest EMS Tower since 1993. Harvard Forest Data Archive: HF069 (v.36). (2021). Environmental Data Initiative: https://doi.org/10.6073/pasta/5c2f17c295413da2a2a091fd7696af40.

27. Foster, D., Barker Plotkin, A. A., Lyford, W. Lyford Mapped Tree Plot at Harvard Forest since 1969. Harvard Forest Data Archive: HF032 (v.20). (2017). Environmental Data Initiative: https://doi.org/10.6073/pasta/292e47940d0b0b07c3be7e9026c12c66.

28. Battles, J. J., Cleavitt, N., Johnson, C., Hamburg, S., Fahey, T., Driscoll, C., Likens, G. Forest Inventory of a Northern Hardwood Forest: Watershed 6, 2017, Hubbard Brook Experimental Forest ver 1. Environmental Data Initiative (2019). (https://doi.org/10.6073/pasta/0593ba15fb76a4f085797126a1bea3a7 (Accessed 2021-10-02).

29. Robertson, G. P., Hamilton, S. K. Long-term ecological research at the Kellogg Biological Station LTER site. The ecology of agricultural landscapes: Long-term research on the path to sustainability, 1-32 (2015).

30. Bourg, N. A., McShea, W. J., Thompson, J. R., McGarvey, J. C., Shen, X. Initial census, woody seedling, seed rain, and stand structure data for the SCBI SIGEO Large Forest Dynamics Plot. Ecology. 94, 2111-2112 (2013). 
31. Johnson, D. J., Magee, L., Pandit, K., Bourdon, J., Broadbent, E., Glenn, K., Kaddoura, Y., Machado, S., Nieves, J., Wilkinson, B., Zambrano, A., Bohlman, S. Canopy tree density and species influence tree regeneration patterns and woody species diversity in a longleaf pine forest. Forest Ecol. and Manag.490, 119082 ISSN 0378-1127 (2021).

32. Pretzsch, H. Forest Diversity and Function: Temperate and Boreal Systems, SchererLorenzen, M. ,Körner, C., Schulze, E.-D., Eds. (Springer Berlin Heidelberg, Berlin, Heidelberg, 2005; https://doi.org/10.1007/3-540-26599-6_3), pp. 41-64.

10

33. Gilbert, G. S., Howard, E., Ayala-Orozco, B., Bonilla-Moheno, M., Cummings, J., Langridge, S., Parker, I. M., Pasari, J., Schweizer, D., Swope, S. Beyond the tropics: forest structure in a temperate forest mapped plot. J. Veg. Sci. 21, 388-405 (2010).

34. Newman, E. A., Wilber, M. Q., Kopper, K. E., Moritz, M. A., Falk, D., McKenzie, D., Harte, J. Disturbance macroecology: a comparative study of community structure metrics in a high-severity disturbance regime, Ecosphere 11(1), e03022 (2020).

35. Newman, E. A., Harte, M. E., Lowell, N., Wilber, M., Harte, J. Empirical tests of withinand across-species energetics in a diverse plant community. Ecology 95(10), 2815-2825 (2014).

36. Gruner, D. S. Geological age, ecosystem development, and local resource constraints on arthropod community structure in the Hawaiian Islands. Biol. J. Linn. Soc. Lond. 90, 551-570 25 (2007). 
37. Hubbell, S. Neutral theory and the evolution of ecological equivalence. Ecology 87, 13871398 (2006).

38. Franzman, J., Brush, M., Umemura, K., Ray, C., Blonder, B., Harte, J. Shifting macroecological patterns and static theory failure in a stressed alpine plant community". Ecosphere 12.6 (2021). DOI: 10.22541/au.159527004.40155464

39. Glazier, D. S. A unifying explanation for diverse metabolic scaling in animals and plants. Biol. Rev. Camb. Philos. Soc. 85, 111-138 (2010).

10

40. Dodds, P. S., Rothman, D. H., Weitz, J. S. Re-examination of the "3/4-law" of metabolism. arXiv [physics.bio-ph] (2000). (http://arxiv.org/abs/physics/0007096).

41. Marquet, P. A., Allen, A. P., Brown, J. H., Dunne, J. A., Enquist, B. J., Gillooly, J. F., Gowaty, P. A., Green, J. L., Harte, J., Hubbell, S. P., O’Dwyer, J., Okie, J. G., Ostling, A., Ritchie, M., Storch, D., West, G. B. On theory in ecology. Bioscience. 64, 701-710 (2014).

42. Golan, A. Foundations of Info-Metrics: Modeling, Inference, and Imperfect Information. Oxford University Press, Oxford UK (2018) (https://play.google.com/store/books/details?id=wMU2DwAAQBAJ).

43. Williams, R.J. Simple MaxEnt models explain food web degree distributions. Theor. Ecol. 3, 45-52 (2010).

44. Payrato-Borras, C., Hernandez, C.L., Moreno, Y. Breaking the spell of nestedness: The entropic origin of nestedness in mutualistic systems. Phys. Rev. X 9, 031024 (2019). 


\section{Acknowledgments:}

We acknowledge the large number of scientists, field technicians, and funding agencies responsible for the data analyzed in this article. Detailed acknowledgements are available in Supplementary Text to this article. JH thanks the Santa Fe Institute and The Rocky Mountain Biological Laboratory for logistic support and useful conversations.

\section{Funding:}

Funding for this research was provided by grant DEB 1753180 to JH from the US National Science Foundation.

Author contributions:

Conceptualization: JH

Development: JH, MB, KU, EAN

Data Curation and Management: EAN

Visualization: MB

Writing: JH, MB, EAN, KU

Competing interests: The authors declare that they have no competing interests.

Data and materials availability: Data sources and permissions are detailed in the main text and supplementary materials. Datasets used in these analyses are available publicly, except Traunstein Large Forest Dynamics Plot, Kellogg Biological Station, and Hawaii arthropod data, which were made available by permission of the data owners and can be requested directly from them. Analysis code for the ecological equation of state scaling is available as Python code from github.com/micbru/equation_of_state/. Data cleaning scripts are available on request from the authors. 


\section{Supplementary Materials}

Materials and Methods

Supplementary Text

Appendices 1-3

5 Figs. S1-S4

Tables S1, S2

\section{Supplementary Video}

Caption

$10 \quad$ Video 


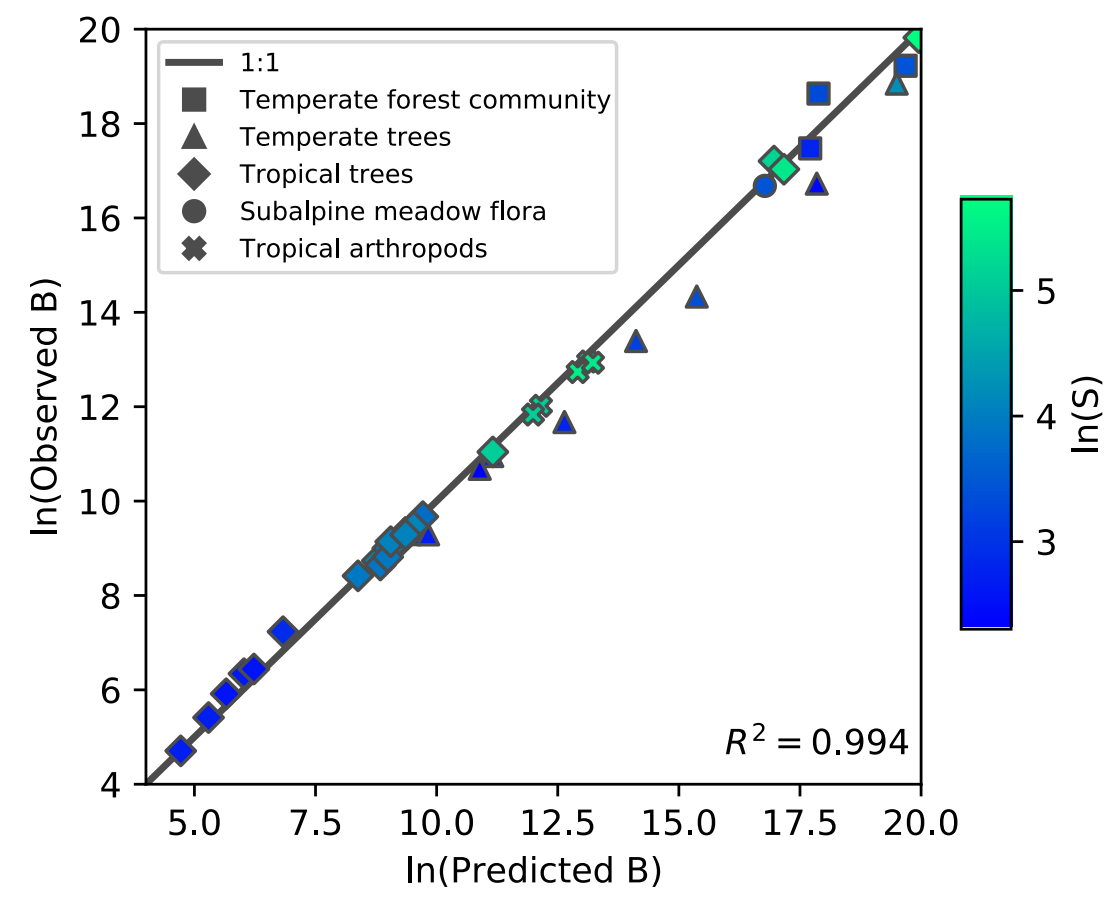

Fig. 1. A test of the ecological equation of state. Observed biomass is determined by either summing empirical masses of individuals or summing empirical metabolic rates raised to the $3 / 4$ power, of each individual. Predicted biomass is determined from Eq. 1 using observed values of $S, N$ and $E$. The quantity $\ln$ (predicted biomass) explains $99.4 \%$ of the variance in observed biomass. Units of mass and metabolism are chosen such that the mass of the smallest individuals are set to 1 and those individuals are also assigned a metabolic rate of 1 . The shape of the marker indicates the type of data, and lighter color corresponds to higher species richness. Data for all analyses come from tropical trees ${ }^{18-25}$, temperate trees ${ }^{26-32}$, temperate forest communities ${ }^{33-34}$, subalpine meadow flora ${ }^{35}$ and tropical island arthropods ${ }^{36}$. 


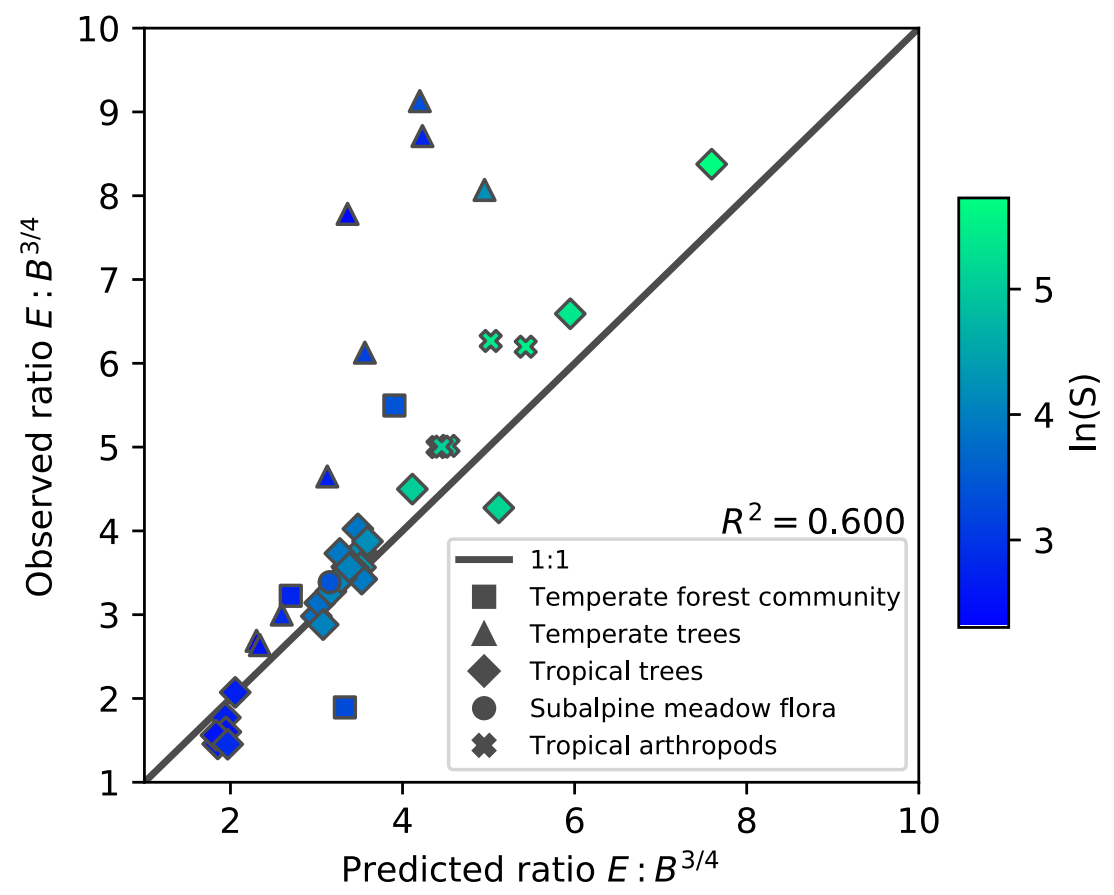

Fig. 2. The explanatory power of diversity and abundance. The observed ratio $E / B^{3 / 4}$ is plotted against the ratio predicted by Eq. 1 . Of the 4-fold variability across ecosystems in that ratio, $60 \%$ is explained by the variability in the predicted combination of diversity and abundance. The shape of the marker indicates the type of data, and lighter color corresponds to higher species richness. Data for all analyses come from tropical trees ${ }^{18-25}$, temperate trees ${ }^{26-32}$, temperate forest communities ${ }^{33-34}$, subalpine meadow flora ${ }^{35}$ and tropical island arthropods ${ }^{36}$. 


\section{Supplementary Files}

This is a list of supplementary files associated with this preprint. Click to download.

- EquationofStateSuppMatNatureHarteetal.pdf

- SupplementaryVideo1.mp4

- Videocaption.pdf 